REVIEW

\title{
IS THERE A LINK BETWEEN ABDOMINAL SURGERY AND OCULAR PRESSURE?
}

\author{
Roxana Chiș $^{1,2}$, Adriana Sîrbu ${ }^{1}$, O. Andronic ${ }^{1,3}$, S. Oprescu ${ }^{1,3}$ \\ ${ }^{1}$ University of Medicine and Pharmacy "Carol Davila" Bucharest, Romania \\ ${ }^{2}$ Clinical Hospital for Ophthalmological Emergencies Bucharest, Romania \\ ${ }^{3}$ Emergency University Hospital of Bucharest, Romania \\ Corresponding author: Chiș Roxana Gabriela \\ E-mail: chisroxana@yahoo.com
}

\begin{abstract}
The mechanisms of variation of intraocular pressure (IOP) during abdominal surgical interventions it is not completely clear and its result on the recovering patient may vary from benign alterations to disabilities. This review aims to identify the most important triggers and its impact of IOP along with individual risk factors during the perioperative period of abdominal surgeries. International literature is providing limited data on this topic. We reviewed 9 studies using PubMed, Scopus, and Web of Science as database. From the multitude of effects and implications that abdominal surgery has onto the physiology of eyeball, the highest impact was linked to the positioning of the patient, induction of anesthesia, perianesthetic events such as intubation and extubation, type of anesthesia and anesthetic used, pneumoperitoneum induced in laparoscopic surgeries and its contribution to the alterations of ocular parameters which are increasing the risk of ocular events. In addition to this, the association of individual risk factors as well as the pre-existing of ophthalmologic diseases may have severe consequences onto the visual function.
\end{abstract}

Keywords: intraocular pressure, abdominal surgery, POVL, acute angle closure

\section{Introduction}

In the current context of medicine on a global level, in which we observe a paradigm shift of medical professions with a narrowing spectrum of each specialization and its pathologies, new niche specializations and overspecializations are developing. We intend to pursue a practical applied issue, but at the same time fundamental as there are limited number of data available in the literature, about the variation of intraocular pressure (IOP) in connection with abdominal surgery.

Outwardly without a clear connection between ophthalmology and general surgery, from the pathophysiological point of view, it is well known that the human body is operating in accordance with the concept of a gear drive, in which the modifications or alterations of a link results in locally and remotely effects. Oftentimes, the impacts cannot be easily quantified or observed, which certifies the fact that the human body is an integrated whole.

Limited data identified on this topic confirms an alteration of IOP during the perioperative period of time noticed among the patients admitted to the general surgery department. Although this theory is little-studied we consider a necessity of knowledge enhancement in an attempt of identifying and emphasizing a possible determinism between surgical cure of abdominal pathologies and the 
occurrence of eye abnormalities (from acute rising of IOP to the acute iridocorneal angle closure).

This review aims to highlight the strong link between two apparently different medical specialties, as well as the impact and multitude of effects that abdominal surgical interventions in its entire ensemble (from the positioning of the patient and surgical technique to the type of anesthesia used) over visual analyzer. Identification and understanding of these issues are leading to an optimal management of the patient, by adopting prophylactic or curative attitudes in a framework of multidisciplinary which forms the desideratum of modern medicine.

\section{Materials and Methods}

For accomplishing this review, we enlisted a throughout research of database as PubMed, Scopus, and Web of Science using key words as "abdominal surgery", "intraocular pressure", and "laparoscopic surgery and eye". Of the very few existing studies, we reviewed the data of 9 studies, the most relevant for the approached theme in this article and from which we concluded this narrative review.

\section{Results}

A number of studies proved the effects of perioperative period of time of abdominal surgical interventions over the eye. It has been analyzed the type of surgery, laparoscopic surgery being the most important, the positioning of the patient during surgery and the type of anesthesia used and its direct effects on IOP. Bearing in mind the physiology and pathophysiology of the entire human body, we consider rightful to embed all these parameters taking in consideration the cumulative risk that each patient has during surgical intervention.

The study of Grosso A. is based on uncertainty linked to etiology and pathology of the increasing IOP during laparoscopic surgery, since it is not clear if the pneumoperitoneum is the main responsible cause of the IOP increase or the association of it with the Trendelenburg position. In this study patients were split in two groups based on their positioning: Trendelenburg or non-Trendelenburg. A bigger increase was observed at patients in Trendelenburg position, of $5.05 \mathrm{mmHg}$ in comparison to the second group in which the growth was of $4.1 \mathrm{mmHg}$. No significant differences were found between the two groups after 48 hours since the intervention. The study concluded that standard pneumoperitoneum, $\leq 14 \mathrm{mmHg}$ produces a moderate and reversible growth of IOP with a tendency of increase when Trendelenburg position is associated [1].

Kaur G. begins his study from the assumption according to which laparoscopic surgery with gas insufflation in the abdominal cavity in Trendelenburg position, may result in severe complications to the optic nerve especially to the susceptible patients. It follows the effects of propofol-based total intravenous anesthesia (TIVA) and sevoflurane anesthesia on IOP comparatively. It concludes that TIVA is more efficient in counteracting the growth of IOP produced by the laparoscopic intervention in Trendelenburg position compared to inhalation anesthesia with sevoflurane, pleading for further studies in case of elder patients or those with preexisting ocular pathologies. [2]

Other studies have reviewed the variation of IOP induced by anesthesia and perianesthesia factors, which can produce the acute iridocorneal angle closure in association with intraocular hypertension. Yukie N. provides in his study a series of individual risk factors, predisposing the patients to developing this pathology, such as: female gender, genetic inheritance, abnormalities or ocular anatomic variations, history of glaucoma and old age. The coexistence of individual risk factors among with intraocular hypertension may lead to the acute closure of iridocorneal angle. Theoretically speaking, any patient has the risk of developing this acute affliction immediately after surgery with general anesthesia.

Therefore, the author of the study considers very important an ophthalmic examination for the purpose of establishing this diagnose, but also the institution of a prompt treatment, for the patients presenting postoperative symptoms such as ocular pain, a reduction in visual acuity unilateral or bilateral, conjunctival hyperemia, cephalalgia or nausea, with the mention that some symptoms may be disguised by 
postoperative administration of painkillers or attributed to general anesthesia. The predictability of occurrence in case of acute angle closure after general anesthesia is low and the preventive measures have not been generally established yet [3]. Notwithstanding the majority of anesthetics are associated with intraocular hypotension, there are a series of factors presented in Yukie N.'s study which lead to the increase of IOP perianesthetic: endotracheal intubation, extubation, hypoxia, hypertension spikes and mydriasis.

Mydriasis represents by far the most important factor involved in the pathology of increased IOP during a surgical intervention with general anesthesia. Mydriasis produces the direct closure of the iridocorneal angle, blocking the draining of aqueous humor through trabecular meshwork by iridotrabecular contact which results in the acute of IOP $[3,4]$.

It is well-known the fact that manipulation of airways is associated with elevated IOP due to the increasing blood pressure, which leads to a growth of eye blood flow. The majority of studies point out a markedly increased of IOP during intubation [5, 6]. In contrast, studies such as R. Madan's pays close attention to the extubation maneuver, less studied in connection with IOP growth, although it reveals a greater increase compared to intubation [6].

Patients with glaucoma are presenting higher growth of IOP compared to non-glaucoma patients both at intubation as well as extubation; they are also predisposed to ocular lesions. Deep anesthesia may reduce IOP up to $15 \mathrm{mmHg}$. Extubation during deep anesthesia may prevent the elevation of IOP at patients with glaucoma. The risks of extubation during deep anesthesia are well-known and include obstruction of upper airways, laryngospasm and pulmonary aspiration. Nonetheless, there are reports which suggest that extubation of an awake patient does not produce any substantial hemodynamic response distinct from extubation under deep anesthesia. However, cough that occurs during conscious extubation may result in an increased IOP of over $50 \mathrm{mmHg}$ and should be avoided in glaucoma patients [6].

R. Madan's study came to the conclusion that glaucoma patients develop a higher IOP compared to normal patients during intubation. Furthermore, extubation led to higher values of
IOP to both groups, glaucoma and non-glaucoma patients, which proved that glaucoma patients have a higher risk of developing ocular impairment or even perioperative visual loss (POVL) [7], although the responses of both groups were similar. He successfully submitted additional information to Kaur G.'s study.

According to the data collected from Bonnie Lee Molloy's study, facial and orbital edema which occurs during some surgical interventions using general anesthesia and Trendelenburg position, as well as the rise of IOP, have resulted in the assumption under which the autoregulation circulatory system of eye and brain are unable to prevent the increase of IOP and the decrease of ocular perfusion pressure (OPP), which represents the difference between IOP and mean arterial pressure (MAP) [8].

Cheng et al studied the alteration of IOP in dorsal decubitus versus ventral decubitus, under general anesthesia from which he observed an increment of almost double in case of the patients positioned in ventral decubitus. The author started his study from the hypothesis according to which if the value of IOP exceeds the value of MAP, during surgical intervention in Trendelenburg position (more than 30 degrees with lower limb elevated), the instituted hypoperfusion may lead to optic nerve ischemia [9]. Moreover, Bonnie Lee Molloy came to the conclusion that there is an existing direct correlation between the duration of surgical intervention in Trendelenburg position and growth of IOP, which leads to a decrease of OPP (sometimes lower than IOP). A sustained decrease of OPP may cause hypoperfusion resulting in the obstruction of blood flow and occurrence of "ophthalmic compartment syndrome" which confer a high risk for development of perioperative visual loss (POVL) [8]

\section{Conclusion}

There are a multitude of effects and implications that abdominal surgery has onto the physiology of the eye. The presented studies show that the entire events surrounding the surgical procedure are inducing alterations whose severity varies from benign to disability. 
Considering the fact that every step of a surgical intervention, including positioning of the patient, induction of anesthesia, perianesthetic events such as intubation and extubation, type of anesthesia and anesthetic used, induced pneumoperitoneum in laparoscopic surgeries and its contribution to the alterations of ocular parameters, increases the risk of ocular events. The association of individual risk factors as well as the pre-existing of ophthalmologic diseases may have severe consequences against the visual function.

\section{References}

[1] Grosso, A., Scozzari, G., Bert, F. et al. Surg Endosc (2013) 27: 3370. https://doi.org/10.1007/s00464-013-2919-2

[2] Kaur, G., Sharma, M., Kalra, P., Purohit, S., \& Chauhan, K. (2018). Intraocular Pressure Changes during Laparoscopic Surgery in Trendelenburg Position in Patients Anesthetized with Propofolbased Total Intravenous Anesthesia Compared to Sevoflurane Anesthesia: A Comparative Study. Anesthesia, essays and researches, 12(1), 67-72. doi:10.4103/aer.AER_177_17

[3] Nitta, Y., Kamekura, N., Takuma, S., \& Fujisawa, T. (2014). Acute angle-closure glaucoma after general anesthesia for bone grafting. Anesthesia progress, 61(4), 162-164. doi:10.2344/0003-300661.4.162

[4] Fazio DT, Bateman JB, Christensen RE. Acute Angle-Closure Glaucoma Associated With Surgical Anesthesia. Arch Ophthalmol. 1985;103(3):360-362. doi:https://doi.org/10.1001/archopht.1985.01050030 056021

[5] Abdulla W, Flaifil H (1992) Intraocular pressure changes in response to endotracheal intubation facilitated by atracurium or succinylcholine with or without lidocaine. Acta Anaesthesiol Belg 43(2):91101

[6] Madan R, Tamilselvan P, Sadhasivam S, Shende D, Gupta V, Kaul HL. Intra-ocular pressure and haemodynamic changes after tracheal intubation and extubation: a comparative study in glaucomatous and nonglaucomatous children. Anaesthesia. 2000;55:380-384

[7] Roth S. (2009). Perioperative visual loss: what do we know, what can we do?. British journal of anaesthesia, 103 Suppl 1(Suppl 1), i31-i40. doi:10.1093/bja/aep295

[8] Molloy, Bonnie Lee. Implications for Postoperative Visual Loss: Steep Trendelenburg Position and Effects on Intraocular Pressure. AANA Journal . Apr2011, Vol. 79 Issue 2, p115-121. 7p. 2 Charts, 2 Graphs

[9] Cheng M, Todorov A, Templehoff R, McHugh T, Crowder CM, Lauryssen C. The effect of prone positioning on intraocular pressure in anesthetized patients. Anesthesiology. 2001;95(6):1351-1355 